\title{
Esquema terapêutico e consumo alimentar em pessoas vivendo com HIV/Aids
}

\author{
Therapeutical scheme and food consumption in people living with HIV/Aids \\ Esquema terapéutico y consumo alimentar en personas viviendo con VIH/AIDS \\ Maria Devany PEREIRA \\ Nayra do Socorro Caldas Carvalho de Almeida TEIXEIRA \\ Iara Katrynne Fonsêca OLIVEIRA \\ Carlos Henrique Ribeiro LIMA \\ Adriana de Azevedo PAIVA \\ Departamento de Nutrição, Universidade Federal do Piauí (UFPI), Campus Universitário Ministro Petrônio Portella, Centro de Ciências de Saúde (CCS) \\ 64049-550 Teresina $-P I$
}

\begin{abstract}
Resumo
Introdução: O HIV é o vírus responsável por destruir os linfócitos T CD4+, sua evolução ocorre a partir de distúrbios que causam infecções oportunistas, alterações nutricionais e até a morte. Uma infecção sexualmente transmissível que atualmente ainda acomete milhares de pessoas no Brasil e no mundo, independente de sexo, raça e condição econômica. A terapia antirretroviral prolonga a vida de Pessoas Vivendo com HIV/Aids diminuindo também a chance de transmissão. Atenção nutricional a esse público é essencial, devido alterações fisiológicas que interferem desde a mastigação até a absorção, assim como interações medicamentosas. Objetivo: Elencar a relação entre o esquema terapêutico e o consumo alimentar de pessoas vivendo com HIV/Aids. Material e Método: Trata-se de um estudo transversal com 42 pacientes diagnosticados com HIV/Aids, de ambos os sexos, com idade superior a 20 anos em tratamento antirretroviral em um hospital de referência de Teresina Piaú, que atendessem os critérios de elegibilidade. Os dados foram coletados a partir de questionário socioeconômico e demográfico, aspectos clínicos e da terapêutica e consumo alimentar, a partir do Recordatório de 24 horas. Resultados e Conclusão: A maioria faz uso do esquema " $3 \mathrm{em} \mathrm{1",} \mathrm{Tenofovir,} \mathrm{lamivudina} \mathrm{e} \mathrm{efavirenz.} \mathrm{E} \mathrm{a} \mathrm{ingestão} \mathrm{média} \mathrm{energética} \mathrm{e} \mathrm{macronutrientes} \mathrm{da}$ população da pesquisa encontram-se dentro dos limites referido pelas DRIs (2005), não foi possível estabelecer relação entre os esquemas antirretrovirais e o consumo alimentar. Isto posto destacamos a necessidade de mais estudos com este público em tratamento antirretroviral quanto à relação do consumo, padrão e perfil alimentar.
\end{abstract}

Descritores: HIV; Terapia Antirretroviral de Alta Atividade; Consumo de Alimentos.

\begin{abstract}
Introduction: HIV is the virus responsible for destroying CD4 + T lymphocytes, its evolution occurs from disorders that cause opportunistic infections, nutritional changes and even death. The infection sexually transmitted that currently affects thousands of people in Brazil and the world, regardless of sex, race and economic condition. Antiretroviral therapy extends the lives of people living with HIV / Aids, also decreasing the chance of transmission. Nutritional attention to this audience is essential, due to physiological changes that interfere from chewing to absorption, as well as drug interactions. Objective: To establish the relationship between the therapeutic scheme and the food consumption of people living with HIV / Aids. Material and Methods: This was a cross-sectional study of 42 HIV / AIDS patients of both sexes, aged over 20 years in antiretroviral treatment at a referral hospital in Teresina Piauí, who met the eligibility criteria. The data were collected from a socioeconomic and demographic questionnaire, clinical and therapeutic aspects and food consumption, from the 24-hour Reminder. Results and Conclusion: Most use the "3 in 1" regimen, Tenofovir, lamivudine and efavirenz. And the average energetic and macronutrient intake of the research population is within the limits reported by DRIs (2005), it was not possible to establish a relationship between antiretroviral regimens and food consumption. This fact highlights the need for more studies with this public on antiretroviral treatment regarding the relation of consumption, standard and dietary profile.
\end{abstract}

Descriptors: HIV; Antiretroviral Therapy, Highly Active; Food Consumption.

\section{Resumen}

Introducción: El HIV es el responsable de destruir los linfocitos T CD4 +, su evolución ocurre a partir de disturbios que causan infecciones oportunistas, alteraciones nutricionales y hasta la muerte. Una infección sexualmente transmisible que actualmente todavía acomete a miles de personas en Brasil y en el mundo, independientemente de sexo, raza y condición económica. La terapia antirretroviral prolonga la vida de las personas que viven con el VIH / SIDA disminuyendo también la posibilidad de transmisión. La atención nutricional a este público es esencial, debido a alteraciones fisiológicas que interfieren desde la masticación hasta la absorción, así como las interacciones medicamentosas. Objetivo: Elencar la relación entre el esquema terapéutico y el consumo alimentario de personas viviendo con VIH / SIDA. Material y método: Se trata de un estudio transversal con 42 pacientes diagnosticados con VIH / Aids, de ambos sexos, con edad superior a 20 años en tratamiento antirretroviral en un hospital de referencia de Teresina Piauí, que cumplan los criterios de elegibilidad. Los datos fueron recolectados a partir de cuestionario socioeconómico y demográfico, aspectos clínicos y de la terapéutica y consumo alimentario, a partir del Recordatorio de 24 horas. Resultados y Conclusión: La mayoría hace uso del esquema "3 en 1", Tenofovir, lamivudina y efavirenz. Y la ingestión media energética y macronutrientes de la población de la investigación se encuentran dentro de los límites referidos por las DRIs (2005), no fue posible establecer relación entre los esquemas antirretrovirales y el consumo alimentario. Esto puso de relieve la necesidad de más estudios con este público en tratamiento antirretroviral en cuanto a la relación del consumo, patrón y perfil alimentario.

Descriptores: VIH; Terapia Antirretroviral Altamente Activa; Consumo de Alimentos.

\section{INTRODUÇÃO}

O vírus da imunodeficiência humana (HIV Human Immunodeficiency Virus) provoca danos no sistema imunológico, pela infecção de células importantes do organismo e grave supressão. Esse distúrbio caracteriza a Síndrome da Imunodeficiência Adquirida (Aids), que evolui devido uma significativa destruição de linfócitos TCD4 $+{ }^{1,2}$.

Segundo o Programa Conjunto das Nações Unidas sobre HIV/AIDS (UNAIDS) ${ }^{3}$, existem mais de 36,7 milhões de pessoas vivendo com HIV no mundo; no Brasil, em 2016, aproximadamente 830.000 pessoas viviam com o vírus, enquanto que o número de mortes relacionadas no país é de 14.000 . É o país que possui maior número de novas infecções por HIV da América Latina, respondendo por $49 \%$ das novas infecções.

No estado do Piauí, de 2007 a 2017 foram notificados 870 casos no Sistema de Informação de Agravos de Notificação (SINAN) sendo o menor entre os estados da região nordeste. Teresina em 2016 
apareceu com taxa de notificação de 24,9 por 100.000 habitantes, estando em $18^{\circ}$ lugar entre as capitais brasileiras ${ }^{4}$.

Com a introdução da Terapia Antirretroviral (TARV), desde a década de 1990, houve melhora na expectativa de vida dos pacientes, assim como também reduziu o numero de mortes ${ }^{5}$. Em 2016, 21,7 milhões tiveram acesso ao tratamento, as mortes relacionadas a Aids caíram de 1,9 milhão em 2005 para 1 milhão em $2016^{3}$.

No Brasil, 490.000 pessoas vivendo com HIV/Aids (PVHA) estão em tratamento, 60\% do total dos infectados, sendo um dos primeiros países a oferecer o tratamento de forma gratuita a todos os doentes, um modelo de destaque no cenário internacional, em especial pela universalidade do acesso $^{3,6}$.

Com o tratamento a Aids passou a ser considerada uma doença crônica e controlável, proporcionando a elevação na contagem de linfócitos T CD4+ e suprimindo a multiplicação do vírus ${ }^{7}$. Porém, o esquema terapêutico tem um tempo indeterminado, com uso de grandes quantidades de comprimidos ao dia; logo, a má adesão é um obstáculo ao tratamento e acarreta em mal prognóstico, possibilitando surgimento de estirpes virais resistentes ${ }^{8}$.

A TARV é um "coquetel" com vários medicamentos, onde preferencialmente se inicia com a combinação de três antirretrovirais (ARV), dois Inibidores de Transcriptase Reversa Análogos de Nucleosídeo (ITRN/ITRNt) associado a uma classe de antirretroviral Inibidor de Transcriptase Reversa Não Nucleosídeo (ITRNN), Inibidor de Protease (IP) ou Inibidor de Integrase (INI) ${ }^{9}$.

A utilização do esquema de tratamento depende de uma avaliação dos benefícios e riscos individualmente, como da replicação viral e reações adversas observadas em cada paciente ${ }^{10}$. Alguns antirretrovirais podem causar importantes reações adversas se combinados com certos alimentos, por essa razão existem diferentes indicações em relação ao esquema terapêutico, como por exemplo, se ingeridos com alimentos ou em jejum ${ }^{11}$. Observa-se que as interações entre TARV, alimentos e nutrição são importantes tanto na absorção medicamentosa quanto no impacto nutricional nesses indivíduos ${ }^{11,12}$.

Ressaltando que uma alimentação saudável e adequada contribui com a melhora da qualidade de vida, além de permitir ao profissional de saúde compreender melhor algumas condições que ajudam no enfrentamento da doença a partir de uma intervenção nutricional adequada ${ }^{13}$. Buscou-se elencar a relação entre o esquema terapêutico e o consumo alimentar de pessoas vivendo com HIV/Aids atendidas no ambulatório de um hospital da capital do Piauí.
MATERIAL E MÉTODO

- Desenho do estudo

Trata-se de um estudo transversal, parte de uma pesquisa intitulada "Efeito da suplementação com vitamina $\mathrm{D}_{3}$ na carga viral e na recuperação de células TCD4+ em pacientes infectados com HIV em terapia antirretroviral assistidos pelo SUS".

- População, amostra e local do estudo

Foram estudados pacientes com diagnóstico de HIV/Aids de ambos os sexos, com idade superior a 20 anos. Abrange amostra não probabilística, de 42 pacientes, selecionados respeitando os critérios de elegibilidade do estudo: Estar em tratamento com antirretrovirais no IDTNP, com idade acima de 20 anos, mulheres que não estejam grávidas e sejam lactantes. Participaram indivíduos em tratamento com antirretrovirais, acompanhados no Instituto de Doenças Tropicais Natan Portela (IDTNP) situado na cidade de Teresina-PI. Este é de natureza terciária, pertence à rede estadual de saúde e recebe pacientes de todas as localidades do Estado, sendo um centro de referência em infecções sexualmente transmissíveis (IST) e doenças tropicais (DT). O IDTNP oferece assistência a uma média de 10.000 de Pessoas Vivendo com HIV/Aids (PVHA) por ano, provenientes da zona urbana e rural da capital do Estado e cidades do interior, além dos que vem de outros estados, como o Maranhão.

\section{- Coleta de dados}

Os dados foram coletados pela aplicação de um formulário estruturado, com informações de idade (em anos), cor da pele (auto-referida), escolaridade (anos de estudo), estado civil e renda familiar, utilizando a classificação do Censo Demográfico do Instituto Brasileiro de Geografia e Estatística $\mathrm{IBGE}^{14}$ para a determinação da faixa de renda. As informações dos esquemas antirretrovirais, recomendações, horários e acompanhamentos dos medicamentos foram coletados no prontuário clínico do paciente a partir da anamnese clínica realizada pelo médico do IDTNP, utilizando uma ficha adaptada do protocolo padrão do hospital.

Partindo dos esquemas antirretrovirais encontrados nos prontuários, foram agrupados em 4 classes de esquema. O Esquema 1 preconiza Tenofovir, lamivudina e efavirenz (TDF+3TC+EFZ), enquanto o Esquema 2, Tenofovir, lamivudina, Dolutegravir (TDF+3TC+DTG). Para o Esquema 3 estão indicados Lamivudina, Zidovudina e Efavirenz (3TC+AZT+EFZ). Outros esquemas diferenciados também são apontados.

\section{- Consumo alimentar}

Para a investigação da ingestão alimentar foi utilizado questionário Recordatório de 24 horas (R24h), aplicado seguindo os cinco passos do Multiple Pass Method (listagem rápida dos alimentos e horários, revisão da listagem rápida, nomeação das refeições, ciclo de detalhamento, revisão geral) ${ }^{15}$. 
As quantidades de energia, carboidratos, lipídios e proteínas foram determinadas a partir do software Virtual Nutri Plus ${ }^{16}$, sendo inseridos os dados de alimentos e preparações não encontrados no programa de acordo com a seguinte sequência de tabelas de composição química de alimentos: Pinheiro et al. ${ }^{17}$ e Bombem et al. ${ }^{18}$, nesta ordem. Os dados presentes nos rótulos dos alimentos industrializados foram utilizados quando estes não constaram nas tabelas.

\section{- Análise de dados}

Os dados foram primeiramente organizados em planilha (software Microsoft Excel 2010), para realização de análises descritivas das variáveis coletadas. As variáveis foram analisadas pelo estudo de média e desvio-padrão por meio do software SPSS Data Editor - Statistical Package for the Social Sciences (versão 5.0 para Windows). A análise da ingestão de macronutrientes no total de calorias da dieta se baseou na variação aceitável de distribuição de macronutrientes das Dietary Reference Intakes DRI's, especificamente pela Acceptable Macronutrient Distribution Ranges - AMDR, sendo classificados conforme os intervalos percentuais ${ }^{19}$.

\section{- Aspectos éticos}

O Estudo foi realizado conforme a legislação de ética em pesquisa para seres humanos (Resolução 466/12), e foi aprovado pelo Comitê de Ética em Pesquisa da Universidade Federal do Piauí (CEP/UFPI) com parecer de número 2.073.649.

\section{RESULTADOS}

A amostra foi constituída por 42 pessoas vivendo com HIV/Aids (PVHA) em tratamento antirretroviral, pelo tempo de 12 meses, cujos dados foram coletados do prontuário hospitalar do paciente, dos questionários sociodemográficos e do consumo alimentar da pesquisa.

\section{- Dados socioeconômicos e demográficos.}

A amostra foi caracterizada com maioria pessoas do sexo masculino, com 46,5 anos de idade em média, predomínio da cor parda e nível médio de escolaridade. Em se tratando da renda familiar, é possível observar que quase metade da amostra recebe até um salário mínimo (Tabela 1).

\section{- Esquemas terapêuticos.}

A análise do esquema antirretroviral, demonstrou que o "Esquema 1", que trata-se do esquema comumente conhecido como " 3 em 1", foi o esquema mais indicado para mais pessoas. O "Esquema 2" em segundo lugar e o "Esquema 3" com menos indicações, o "Outros" agrupou os 36\% dos participantes da pesquisa que fazem uso de esquemas diferenciados (Gráfico 1). Observa-se que dentre os 42 esquemas, o medicamento Lamivudina (3TC) está em quase todos os esquemas.

\section{- Recordatório alimentar}

Em relação à ingestão energética diária e macronutrientes dos entrevistados (média e desvio padrão) podem ser observados na Tabela 2, assim como a porcentagem de proteínas, carboidrato e lipídios, de acordo com o Valor Calórico Total (VCT).

De acordo com a referência das $\mathrm{AMDR}^{20}$ que estão entre $45-65 \%$ para carboidratos, 10 a $35 \%$ de proteínas e 20 a $35 \%$ de lipídios, os valores encontrados para o sexo masculino estão dentro das recomendações, em relação ao sexo feminino apenas os valores de gordura total estão acima do recomendado.

Tabela 1. Caracterização do perfil socioeconômico de PVHA atendidas em ambulatório hospitalar, Teresina-PI, 2018

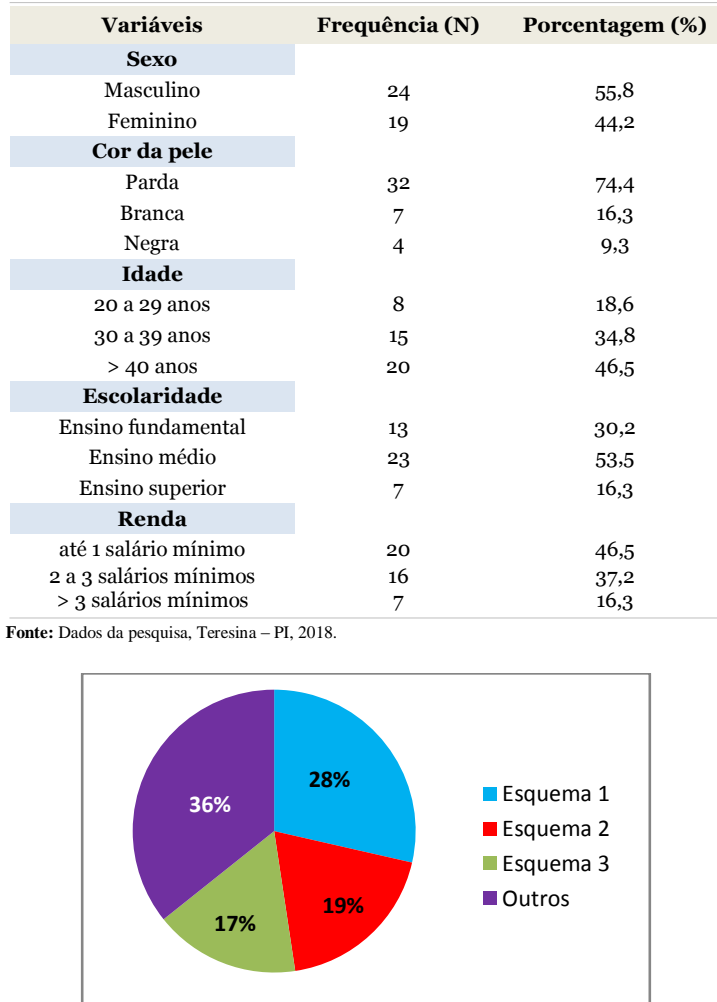

Gráfico 1. Esquemas antirretrovirais de PVHA atendidas em ambulatório hospitalar, Teresina-PI, 2018

Tabela 2. Ingestão energética e consumo de macronutrientes em PVHA atendidas em ambulatório hospitalar, Teresina-PI, 2018

\begin{tabular}{|ccccccc}
\hline Sexo & \multicolumn{3}{c}{ Feminino } & \multicolumn{3}{c}{ Masculino } \\
Média & DP & \% & Média & DP & \% \\
Energia (Kcal) & 1490 & 731,9 & - & 2113,3 & 755,1 & - \\
Proteína (g) & 67,3 & 33,6 & $18 \%$ & 102,3 & 41,6 & $19 \%$ \\
Carboidrato (g) & 191,1 & 95,5 & $51 \%$ & 273,5 & 107,7 & $51 \%$ \\
Lipídio (g) & 62,4 & 160,3 & $37 \%$ & 68,0 & 35,6 & $28,9 \%$ \\
\hline
\end{tabular}

- Relação entre o esquema terapêutico, ingestão energética e consumo de macronutrientes

A Tabela 3 mostra a diferença de média de nutrientes segundo os esquemas terapêuticos. Ao aplicar-se o teste de diferença de média do consumo de energia, proteína, carboidrato e lipídio com o esquema terapêutico, pode-se inferir que não houve diferença estatisticamente significativa para nenhuma das variáveis.

Observa-se que os valores de energia e carboidratos são maiores em indivíduos que fazem 
uso do "Esquema 1", enquanto os pacientes com uso do "Esquema 2" apresentou um consumo maior de lipídio e os do "Esquema 3" um consumo maior de proteínas, quando comparado com os outros esquemas.

Tabela 3 - Média e desvio padrão de energia e macronutrientes segundo o esquema terapêutico em PVHA atendidas em ambulatório hospitalar, Teresina-PI, 2018.

\begin{tabular}{cccccc}
\hline \multicolumn{2}{c}{$\begin{array}{c}\text { Esquema } \\
\text { terapêutico }\end{array}$} & Energia (Kcal) & Proteína (g) & Carboidrato (g) & Lipídio (g) \\
Esquema 1 & Média & 2058,82 & 87,16 & 282,54 & 66,67 \\
& DP & 652,98 & 23,58 & 117,17 & 32,33 \\
Esquema 2 & Média & 1898,12 & 96,50 & 257,0 & 142,35 \\
& DP & 673,63 & 27,49 & 97,23 & 233,85 \\
Esquema 3 & Média & 2041,14 & 100,28 & 237,42 & 77,28 \\
& DP & 840,64 & 53,75 & 84,51 & 44,01 \\
Outros & Média & 1557,60 & 76,25 & 193,13 & 51,94 \\
& DP & 927,16 & 53,06 & 111,63 & 38,57 \\
Total & Média & 1846,26 & 87,23 & 238,22 & 77,59 \\
Valor de p & DP & 799,71 & 41,86 & 109,48 & 107,25 \\
Fonte: Dados da pesquisa, Teresina - PI, 2018. & 0,567 & 0,192 & 0,192 \\
\hline
\end{tabular}

\section{DISCUSSÃO}

Do ano 2000 a junho de 2018, registrou-se um total de 717.318 casos de Aids, sendo que $507.890(70,8 \%)$ foram notificados no SINAN. A região Nordeste foi uma que apresentou tendência de crescimento. Em 2007 a taxa registrada desta região foi de 12,7 casos por 100 mil habitantes, enquanto em 2017 foi de 15,7 representando aumento de $24,1 \%{ }^{4}$.

Segundo dados do Ministério da Saúde até 2017, 67,9\% dos casos notificados foram do sexo masculino e apenas $32,1 \%$, do sexo feminino, corroborando com os dados do presente estudo ${ }^{9}$. Em semelhante, apresentado por Ladeira e Silva ${ }^{20}$, a maioria (59\%) das PVHA era do sexo masculino.

Dentre as mudanças no perfil da doença, também deixou de ser restrito ao grupo de pessoas que fazem sexo com pessoas do mesmo sexo, como era relacionado antigamente. O SINAN mostra um aumento na proporção de casos decorrentes da exposição homossexual nos últimos anos, que passou de 35,6\% em 2006 para 48,9\% em 2017, porém os valores em indivíduos com exposição heterossexual ainda é alto com $37,6 \% 4$.

Outro fator é que o nível de escolaridade, ainda é importante, pois influencia tanto na infecção por HIV/Aids como na evolução clínica da doença, devido a falta de acesso a informações, métodos de prevenção e compreensão do impacto positivo do tratamento ${ }^{21}$.

Em relação à cor da pele, o estudo epidemiológico de Galvão et al. ${ }^{22}$ em um município do Maranhão, assemelha-se ao nosso, onde ao relacionando à raça/cor, mostrou que entre os registros de 2005 a 2014, os pardos $213(54,6 \%)$ predominaram, logo em seguida, a cor negra 78 (20\%). Nas notificações do SINAN, em relação à cor no período de 2007 a 2017, 47,6\% eram brancos e $51,5 \%$ eram pretos e pardos ${ }^{9}$. Ressaltamos que a notificação da infecção pelo HIV ainda é muito recente no país, o que impede uma analise epidemiológica rigorosa com relação às tendências da infecção no Brasil ${ }^{4}$.

Quanto ao esquema antirretroviral das PVHA do estudo, percebe-se que a variedade de esquema encontrado poderá ter relação com os vários médicos do ambulatório hospitalar, mesmo atualmente buscarem padronizar levando em conta o protocolo indicado pelo Ministério da Saúde. Algumas mudanças são utilizadas a partir das individualidades e particularidades dos pacientes, como os efeitos colaterais ou resistência a algum medicamento. Observou-se também que as recomendações médicas limitam-se aos horários de tomada destes.

No estudo de Furini et al. ${ }^{23}$ o esquema mais empregado foi o composto por darunavir, tenofovir, lamivudina, raltegravir e ritonavirsendo $(37,5 \%)$ destes, $90 \%$ utilizaram o darunavir e ritonavir em combinações com outros antirretrovirais. Porém, os esquemas sofrem mudanças constantes e rápidas, no mesmo estudo de Furini et al. ${ }^{23}$ os esquemas eram com maiores quantidade de cápsulas comparadas com o atual, onde dois ou três medicamentos pode ser o suficiente para o tratamento.

Segundo o Protocolo Clínico e Diretrizes Terapêuticas para Manejo da Infecção pelo HIV de 2015, como regra, o esquema de terapia inicial deve ser o "3 em 1" (Tenofovir, Lamivudina e Efavirenz). No ano de 2017 o esquema para inicio do tratamento era preferencialmente o "Tenofovir, Lamivudina e Dolutegravir", deixado como alternativa o esquema de 2015, para quando o Dolutegravir for contraindicado $^{24}$.

Pode-se levantar a explicação devido o dolutegravir apresentar vantagens ao efavirenz, pois este causava, dentre outros sintomas, diarreia, tontura, sonolência, fadiga, dor de cabeça, suores, dificuldade de concentração, insônia ${ }^{25}$. Já o dololutegravir tem alta potência, alta barreira genética, dose única diária e poucos efeitos adversos, garantindo um tratamento mais duradouro $\mathrm{e}$ seguro $^{26,27}$.

Ainda nesta pesquisa foi possível perceber, em sua maioria, a associação do tenofovir + lamivudina (TDF/3TC), conhecido como esquema "2 em 1". Alguns estudos mostraram que estes além de estarem disponíveis em coformulação e permitir tomada única diária, apresenta um perfil favorável em termos de toxicidade, supressão virológica, resposta de LT-CD4+, lipoatrofia e toxicidade hematológica quando comparada ao Zidovudina $(\mathrm{AZT})^{28,29}$.

Sobre o consumo alimentar no presente estudo, pessoas do sexo feminino apresentarem valores acima do referente de gordura total, podendo estar relacionado tanto ao consumo alimentar propriamente dito, como também ao tratamento do HIV. A Atenção especial a esse perfil lipídico se deve em relação a altas taxas de lipodistrofia e 
dislipidemias associados a esses pacientes. Segundo Costa $^{30}$, além dessa redistribuição da gordura corporal (lipodistrofia), também existem alterações nos níveis de colesterol e triglicérides (dislipidemias), comprometendo a saúde física e psíquica do paciente que faz uso da terapia antirretroviral ou não.

$\mathrm{Na}$ análise do consumo alimentar no estudo de Dutra et al. ${ }^{31}$ com PVHA foi evidenciado dietas inadequadas em macro, micronutrientes e com dieta hipercolesterolêmica. Santo et al. ${ }^{32}$ mostrou inadequação calórica de $89 \%$ e proteica de $73,7 \%$ dos pacientes, apresentando ingestão acima do limite superior da faixa de recomendação preconizada na literatura para portadores de HIV assintomáticos.

A mudança dos hábitos alimentares, como consumo excessivo de gorduras e a baixa ingestão de fibras alimentares acabam ocasionando prejuízo à saúde da PVHA, pois no cenário atual a hiperalimentação pode ser tão prejudicial quanto à desnutrição ${ }^{33}$.

No presente estudo, a média de ingestão energética foi adequada, levando em consideração a variação de idade, provavelmente uma segunda aplicação seja necessária para identificação mais precisa do consumo alimentar destes para um entendimento maior e propormos as causas e efeitos da relação do aumento de calorias com o HIV/aids. No estudo de Dutra et al. ${ }^{31}$ foi encontrado em 39 pacientes analisados, a média geral de $2.148,38 \mathrm{Kcal} / \mathrm{dia}$ de calorias ingeridas, em semelhança com a nossa pesquisa.

Mesmo a pesquisa mostrando uma média de consumo energético adequado vale lembrar a importância de uma análise qualitativa destes alimentos, pois mesmo tendo uma redução significativa dos efeitos adversos desses medicamentos, alguns hábitos alimentares estão diretamente relacionados a estes efeitos a curto e longo prazo. Como mostra Brito ${ }^{34}$ em seu guia de cuidados aos pacientes em uso de terapia antirretroviral, condutas de saúde aos principais efeitos adversos aos antirretrovirais, levam ao bem estar do paciente e tratamento mais eficaz.

As PVHA são frequentemente acometidas por desordens nutricionais, devendo levar em consideração também a condição socioeconômica, fator determinante tanto na escolha e consumo dos alimentos, quanto no acompanhamento correto do tratamento, influenciando diretamente na qualidade de vida destas. Na pesquisa de Silva et al. ${ }^{35}$, a desnutrição nesses pacientes muitas vezes foi relacionada a alterações de consumo alimentar errôneas, deglutição deficiente, absorção prejudicada, infecções oportunistas, depressão, além da dificuldade de acesso ao alimento pela precariedade socioeconômica da maioria da população atingida.

E devido a alterações de consumo alimentar errôneas, a escolha do esquema antirretroviral deve levar em consideração as interações potencialmente negativas, que vai desde o horário, quanto o que pode ser consumido com este medicamento. A cartilha do Banco Mundial ${ }^{11}$ sobre HIV/Aids nutrição e segurança alimentar mostra, que o Efavirenz, quando utilizado com refeição pode reduzir a intensidade dos efeitos colaterais, mas não se recomenda uma refeição rica em gordura, que poderá prejudicar sua absorção.

O estudo de Furini et al. ${ }^{23}$, mostrou que dos pacientes estudados o ritonavir, darunavir e o tenofovir, foram os antirretrovirais que mais apresentaram riscos para interações com alimentos, sendo que todos relataram tomar os medicamentos após as refeições. A maioria dos entrevistados do presente estudo fazia uso dos medicamentos pela manhã após o café ou anoite após o jantar, antes de deitar, por indicação médica e algumas vezes por escolha do próprio paciente.

Nesse estudo quando comparados as médias do "Esquema 1" que contém o Efavirenz com o "Esquema 2" que não contém, não foi possível observar associação com o consumo alimentar, mesmo com consumo de gordura total no grupo do "Esquema 2" apresentaram média elevada.

No "Esquema 1", que traz o efavirenz, traz também o tenofovir e lamivudina, onde esses dois são recomendados administrar com ou sem alimento, e dentre alguns efeitos adversos apresentados temos, náuseas, dor abdominal, flatulência, diarreia, dentre outros $^{34}$. Em contrapartida o estudo não mostrou associação negativa do esquema terapêutico com o consumo alimentar desse grupo.

Quando analisado o grupo "outros" que foi a maior proporção de pessoas e trata-se do esquema terapêutico diferenciado para cada PVHA, percebemos que o consumo de energia $(1557,60 \mathrm{kcal})$ foi o menor valor encontrado dentre os quatro grupos, porém a variação de esquemas não nos permite traçar um perfil de associação com os medicamentos.

Uma revisão apresentada por Sousa et al. ${ }^{36}$ demonstra a importância da atenção na terapia juntamente com os nutrientes fornecidos, uma vez que interfere direta e indiretamente na biodisponibilidade podendo gerar falhas na resposta terapêutica. Além das infecções relacionadas com HIV, muitas vezes tem-se o estado nutricional como fator determinante para a incidência e severidade, um acompanhamento nutricional relacionado ao tratamento antirretroviral, além de ser um fator de recuperação do bem estar físico, é também primordial na manutenção da qualidade de vida ${ }^{37}$.

A ausência de recomendações específicas para esse público e a escassez de estudos com descrição da ingestão alimentar e de práticas alimentares corretas para esses pacientes em terapia antirretroviral, limita a discussão de resultados. Ressalvamos aqui a importância da orientação aos 
pacientes com HIV/Aids sobre a melhoria na sua alimentação, estimulando o consumo de alimentos ricos em micronutrientes, fibras e até restrição de alguns tipos de alimentos podem ser necessários para recuperação do quadro clínico/ laboratorial dos pacientes $^{12}$.

$\mathrm{Na}$ coleta de informações sobre o consumo alimentar em um grupo de médio a grande número de participantes, a escolha do R24h é o mais vantajoso, pois sua aplicação é rápida e direta com o entrevistado, ajudando a participação e recordação do mesmo, esse método avalia a dieta atual, estimando valores da ingestão de energia e nutrientes distribuídos no total de alimentos consumidos pelo indivíduo mesmo este ${ }^{38}$. Porém, alguns estudos apontam uma ingestão de energia relatada nas avaliações de consumo alimentar, frequentemente, abaixo do valor real, e embora possa se justificar em partes devido a deficiências nas tabelas de composição de alimentos utilizadas para conversão dos alimentos, a maioria trata-se do autorelato da ingestão ${ }^{39}$.

Portanto, para traçar um perfil dietético mais fidedigno à realidade dessas pessoas, seria necessário um acompanhamento por um período de tempo maior, com a aplicação de outros inquéritos dietéticos com maior acurácia.

\section{CONCLUSÃO}

$\mathrm{O}$ grupo de pessoas vivendo com HIV/Aids atendidas no ambulatório do hospital de referência da capital do Piaú, caracterizou-se pela maioria do sexo masculino, acima dos quarenta anos de idade com estudo até o nível médio e renda de até um salário mínimo. A média de consumo de energia, carboidrato, proteína e lipídios foram adequados e quando comparadas com os diferentes esquemas antirretrovirais, não se apresentaram associados, podendo ser devido ao pequeno número da amostra ou até mesmo as muitas variações dos esquemas utilizados.

Destacamos a necessidade de mais estudos na população vivendo com HIV/Aids em tratamento antirretroviral quanto à relação do consumo, padrão e perfil alimentar uma vez que as carências nutricionais são inerentes ao quadro e requer acompanhamento ao longo de todo o tratamento médico. Dentro desta nossa perspectiva percebemos a necessidade de mudanças e melhoras no tratamento nutricional para a conquista de ganhos na saúde das PVHA.

\section{AGRADECIMENTOS}

À Fundação de Amparo à Pesquisa do Estado do Piauí - FAPEPI - EDITAL FAPEPI/ MSDECIT/CNPq/SESAPI No $N^{\circ} 002 / 2016$ - PPSUS Processo027/2016 pelo suporte financeiro.

\section{REFERÊNCIAS}

1. Reis L. Perfil nutricional de crianças e adolescentes portadores de HIV em acompanhamento ambulatorial [dissertação]. São Paulo: Faculdade de Saúde Pública - USP; 2008.

2. Armentano TC, Silva AR, Ferrari LV, Mateus NG, Mello R. A lipodistrofia em pacientes que vivem com HIV/AIDS. Rev pesqui cuid fundam. 2013;5(5esp):173-81.

3. Joint United Nations Programme on HIV/AIDS (UNAIDS). Global report: UNAIDS report on the global AIDS epidemic. Geneva: WHO Library Cataloguing-in-Publication Data; 2012

4. Ministério da Saúde. Boletim epidemiológico AIDS-DST. Semanas Epidemiológicas. Brasília:Ministério da Saúde 2013.

5. Barros SG, Vieira-da-Silva LM. Terapia antirretroviral combinada, a política de controle da Aids e as transformações do Espaço Aids no Brasil dos anos 1990. Saúde Debate. 2017; 41(esp3):114-28.

6. Tse WF, Yang W, Huang W. A narrative review of cost-effectiveness analysis of people living with HIVtreated with HAART: from interventions to outcomes. Clinicoeconom Outcomes Res. 2015;7:431-39.

7. Braga LA, Silva CAB. Avaliação nutricional e metabólica de pacientes com Hiv em uso da terapia antirretroviral no nordeste do Brasil. RBPS. 2010;23(4):368-73.

8. Freitas JP, Sousa LRM, Cruz MCMA, Caldeira NMVP, Gir E. Terapia com antirretrovirais: grau de adesão e a percepção dos indivíduos com HIV/Aids. Acta paul enferm. 2018;31(3):327-33.

9. Brasil. Ministério da Saúde. Secretaria de Vigilância em Saúde - Departamento de DST, AIDS e Hepatites Virais. Boletim Epidemiológico - AIDS e DST. Brasília (DF), Ano V - $\mathrm{n}^{\circ} 1$, jan. a jun. de 2017.

10.Brasil. Ministério da Saúde. Secretaria de Vigilância em Saúde. Programa Nacional de DST e Aids. Síndrome Lipodistrófica em HIV. Brasília: MS, 2011.

11.Banco Mundial. HIV/SIDA, nutrição e segurança alimentar: o que podemos fazer - uma síntese de princípios de orientação internacionais. Washington DC, Banco Mundial, 2008.

12.Brasil. Ministério da Saúde. Secretaria de Vigilância em Saúde. Programa Nacional de DST/AIDS. Manual clínico de alimentação e nutrição na assistência a adultos infectados pelo HIV / Ministério da Saúde, Secretaria de Vigilância em Saúde, Programa Nacional de DST/Aids. Brasília: Ministério da Saúde, 2006.

13.Brasil. Ministério da Saúde. Secretaria de Vigilância em Saúde. Programa Nacional de DST/AIDS. Alimentação e nutrição para pessoas que vivem com HIV e AIDS / Ministério da Saúde, Secretaria de Vigilância em Saúde, Programa Nacional de DST/AIDS. Brasília: 
Ministério da Saúde, 2006.

14.IBGE. Censo demográfico 2010. IBGE: Instituto Brasileiro de Geografia e Estatística, 2010.

15.Moshfegh AJ, Rhodes DG, Baer DJ, Murayi T, Clemens JC, Rumpler WV et al. The US Department of Agriculture Automated MultiplePass Method reduces bias in the collection of energy intakes. Am J Clin Nutr. 2008;88(2):324-32.

16.Pinheiro ABV, Lacerda EMA, Benzecry EH, Gomes MCS, Costa VM. Tabela para avaliação de consumo alimentar em medidas caseiras. 5. ed. São Paulo: Atheneu; 2005.

17. Bombem KCM, Canella DS, Bandoni DH, Jaime PC. Manual de medidas caseiras e receitas para cálculos dietéticos. In: Manual de medidas caseiras e receitas para cálculos dietéticos. São Paulo: M Books; 2012.

18.Freitas AMP, Philippi ST, Ribeiro, SML. Food lists from the diet of a group of elderly individuals: analysis and perspectives. Rev Bras Epidemiol. 2011;14(1):1-16.

19. Trumbo P, Schlicker S, Yates AA, Poos M, Food and Nutrition Board of the Institute of Medicine, The National Academies. Dietary reference intakes for energy, carbohydrate, fiber, fat, fatty acids, cholesterol, protein and amino acids. J Am Diet Assoc. 2002;102(11):1621-30.

20.Ladeira POC, Silva DCG. Estado Nutricional e perfil alimentar de pacientes assistidos pelo programa de DST/AIDS e hepatites virais de um centro de saúde de Itaperuna-RJ. DST J bras Doenças Sex Transm. 2012;24(1):28-31.

21.Kauffmann LKO, Miranda RNA, Guterresc AS, Pinto AF. Perfil nutricional e alimentar de portadores de HIV-1/AIDS internados em um hospital universitário. Ciência\&Saúde. 2017;10(2):82-8.

22. Galvão JMV, Costa ACM, Jaime VG. Perfil sócio demográfico de portadores de HIV/AIDS de um serviço de atendimento especializado. Rev Enferm UFPI. 2017;6(1):4-8.

23.Furini AAC, Lima TAM, Rodrigues JF, Borges MSB, Carmo, EGB, Cecchim MC et al. Análise de interações medicamentosas e alimentares em pacientes com AIDS em uso da TARV associada à terapia de resgate. Rev Ciênc Farm Básica Apl., 2015;36(3):427-34.

24.Brasil. Ministério da Saúde. Secretaria de Vigilância em Saúde. Protocolo Clínico e Diretrizes Terapêuticas para Manejo da Infecção pelo HIV em Adultos / Ministério da Saúde, Secretaria de Vigilância em Saúde, Departamento de Vigilância, Prevenção e Controle das Infecções Sexualmente Transmissíveis, do HIV/Aids e das Hepatites Virais. Brasília: Ministério da Saúde, 2018.

25.Brasil. Ministério da Saúde. Secretaria de Vigilância em Saúde. Departamento de DST, Aids e Hepatites Virais. Protocolo de assistência farmacêutica em DST/HIV/Aids: recomendações do Grupo de Trabalho de Assistência Farmacêutica. Brasília: Ministério da Saúde, 2010.

26. Walmsley S, Baumgarten A, Berenguer J, Felizarta F, Florence E, Khuong-Josses MA et al. Dolutegravir Plus Abacavir/ Lamivudine for the Treatment of HIV-1 Infection in Antiretroviral Ó erapy-Naive Patients: Week 96 and Week 144 Results From the SINGLE Randomized Clinical Trial. J Acquir Immune Defic Syndr. 2015; 70(5):515-19.

27.Castagna A, Maggiolo F, Penco G, Wright D, Mills A, Grossberg $\mathrm{R}$ et al. Dolutegravir in antiretroviralexperienced patients with raltegravirand/or elvitegravir-resistant HIV-1: 24-week results of the phase III VIKING-3 study. J Infect Dis. 2014;210(3):354-62.

28. Smith KY, Patel P, Fine D, Bello N, Sloan L, Lackey $\mathrm{P}$ et al. Randomized, double-blind, placebo-matched, multicenter trial of abacavir/lamivudine or tenofovir/emtricitabine with lopinavir/ritonavir for initial HIV treatment. AIDS. 2009;23(12):1547-56.

29.Sax PE, Tierney C, Collier AC, Fischl MA, Mollan K, Peeples L et al. Abacavir-lamivudine versus tenofoviremtricitabine for initial HIV-1 therapy. N Engl J Med. 2009;361(23):2230-40.

30.Costa FD. Síndrome lipodistrófica do hiv em pacientes da URE DIPE em Belém do Pará.[dissertação]. Belém: Programa de Mestrado Multidisciplinar em Saúde - UFPA; 2014.

31.Dutra CDT, Salla LCN, Marques MCM, Libonati RMF. Avaliação do consumo alimentar em pacientes HIV positivos com lipodistrofia. Rev Ciênc Saúde. 2011;4(2):59-65.

32. Santo RE, Grinsztejn E, Peres WAF, Brito PD. Bioimpedância e antropometria na determinação da composição corporal em homens portadores de HIV. Rev Bras Nutr Clin. 2016; 31(1):60-4.

33. Silva EFR, Lewi DS, Vedovato GM, Garcia VRS, Tenore SB, Bassichetto KC. Estado nutricional, clínico e padrão alimentar de pessoas vivendo com HIV/Aids em assistência ambulatorial no município de São Paulo. Rev Bras Epidemiol. 2010;13(4):677-88.

34. Brito DMS. Guia de cuidados aos pacientes em uso de terapia antirretroviral. Fortaleza; 2012.

35. Silva MCA, Burgos MGPA, Silva RA. Alterações nutricionais e metabólicas em pacientes com AIDS em uso de terapia anti-retroviral. DST J bras Doenças Sex Transm. 2010; 22(3):118-22.

36. Souza DS, Hora TF, Padilha PC, Gonçalves JCS, Peres WAF. Efeito dos alimentos na biodisponibilidade de medicamentos antirretrovirais: uma revisão sistemática da literatura. Nutrire. 2014;39(2):243-51.

37.Savi LGGFS. Importância da intervenção nutricional em pacientes com Síndrome 
Consumptiva proveniente da Síndrome da Imunodeficiência Adquirida [monografia]. Brasília: Faculdade de Ciências de Educação e Saúde - UNICEUB; 2013.

38. Fisberg RM, Marchioni DML, Colucci ACA. Avaliação do consumo alimentar e da ingestão de nutrientes na prática clínica. Arq Bras Endocrinol Metab. 2009;53(5):617-24.

39. Rocha PB. Perfil alimentar e nutricional dos pacientes HIV positivo atendidos em um serviço público de saúde de Porto Alegre/RS [monografia]. Porto Alegre: Universidade Federal de Rio Grande do Sul; 2007.

\section{CONFLITO DE INTERESSES}

Os autores declaram não haver conflitos de interesse.

AUTOR PARA CORRESPONDENCIA

Iara Katrynne Fonseca Oliveira

iarakatrynne@hotmail.com

Submetido em 07/02/2019

Aceito em 24/05/2019 